\title{
Longitudinal carrier density profiling in semiconductor lasers via spectral analysis of side spontaneous emission
}

\author{
Edward H. Sargent, ${ }^{\text {a) }}$ Dorothy Pavlidis, Hanan Anis, Nicolae Golinescu, and J. M. Xu \\ Department of Electrical and Computer Engineering, University of Toronto, Toronto, Ontario, \\ Canada M5S 1 A4 \\ Richard Clayton and Hyung B. Kim \\ NORTEL Technology, P.O. Box 3511, Station C, Ottawa, Ontario, Canada K1Y $4 H 7$
}

(Received 28 February 1996; accepted for publication 23 April 1996)

\begin{abstract}
A combined experimental and theoretical approach to measuring the variation in carrier density along the length of a semiconductor laser is developed. It is shown that by following the rate of increase of the principal spectral peak, rather than monitoring the optical power at a fixed energy, measurements can be made less susceptible to the effects of heating in the sample. Experimental results showing the development of the longitudinal carrier density profile with injected current are presented and, when compared with the results of self-consistent modeling, provide insights into the internal operating mechanisms of the laser. (C) 1996 American Institute of Physics.

[S0021-8979(96)02415-2]
\end{abstract}

Spontaneous emission from the active region of a semiconductor laser is rich in information, such as the relative strength of radiative transitions in the material, the local field intensity and carrier density, and the temperature. In order to determine these key parameters rigorously and selfconsistently, a number of independent measurements would be required; such an intricate and challenging investigation has yet to be reported. Instead, previous investigators have introduced simplifying assumptions or have neglected suitably small effects, focusing their attention on a regime in which these approximations are valid. For example, frontfacet amplified spontaneous emission has long been used to study net modal gain during passage through the cavity. ${ }^{1,2}$ More recently, detailed analysis of spontaneous emission spectra from a top-contact window has provided valuable insights into higher confined multiquantum-well states, ${ }^{3}$ gain nonlinearity, ${ }^{4,5}$ recombination mechanisms, ${ }^{6}$ and temperature sensitivity of the laser threshold. ${ }^{7}$ The restriction on these techniques is that they can usefully be applied only below the laser threshold - a regime in which longitudinal variations may be neglected.

Progress was recently made in the experimental characterization of longitudinal effects through the study of spontaneous emission over a fixed, narrow wavelength range at a few positions along the length of the laser cavity. ${ }^{8}$ The results of this approach demonstrated the dependence of the degree of carrier nonpinning in the above-threshold regime on longitudinal position.

We sought to combine the merits of these two approaches by studying spatial variations using the techniques of spectral analysis pioneered by the first group of authors. Our approach was two pronged. We began by examining theoretically the relationship between the local carrier density and the salient spectral features of the spontaneous emission. On the basis of this investigation, we formulated an experimental and analytical technique which benefited from

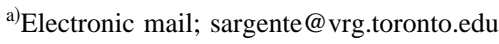

a minimal sensitivity to changes in temperature and which exhibited a simple relationship between the carrier density and the intensity of the first quantum-well transition peak. With this new tool in hand, we embarked on a study of the longitudinal variations in the carrier density profile.

We begin by describing our experimental technique. We measured spontaneous emission from the side of a laser cleaved along its length at a distance of $20 \mu \mathrm{m}$ from the ridge. The shortest resonant cavity in the direction of measurement (formed by the cleaved edge and the ridge) gives rise to oscillations with a period of $0.009 \mathrm{eV}$, an order of magnitude down from the separation of the spectral peaks studied. These Fabry-Pérot oscillations could be filtered out, if necessary, without losing the essential information required for analysis of the spectra. A tapered fiber was translated longitudinally and aligned in the lateral and transverse directions using a micropositioning system. The lasers under study are fabricated from metal-organic chemical-vapordeposition (MOCVD) grown single-quantum-well (SQW) wafers, with no intentional GaAs buffers cladding the QW. The device on which detailed results are reported herein had $10 \%$ reflectivity mirrors deposited on both facets to increase the threshold carrier density. Similar devices with 10\%/90\% coatings had threshold currents around $20 \mathrm{~mA}$.

On the theoretical side we began by calculating band offsets for the strained materials system under study. ${ }^{9}$ The quantized energy levels in the conduction band and the valence band (for both heavy and light holes) were then determined. The difference in energies between the first electronlight-hole transition and the first electron-heavy-hole transition is $0.08 \mathrm{eV}$. Experimental spontaneous emission spectra (Fig. 1) agreed well with these calculations. Also evident in the measured spectra is a decrease in the first transition energy with current, attributable to temperatureinduced band-gap shrinkage. The measured spectra exhibited the anticipated Fabry-Pérot-type oscillations with a constant 


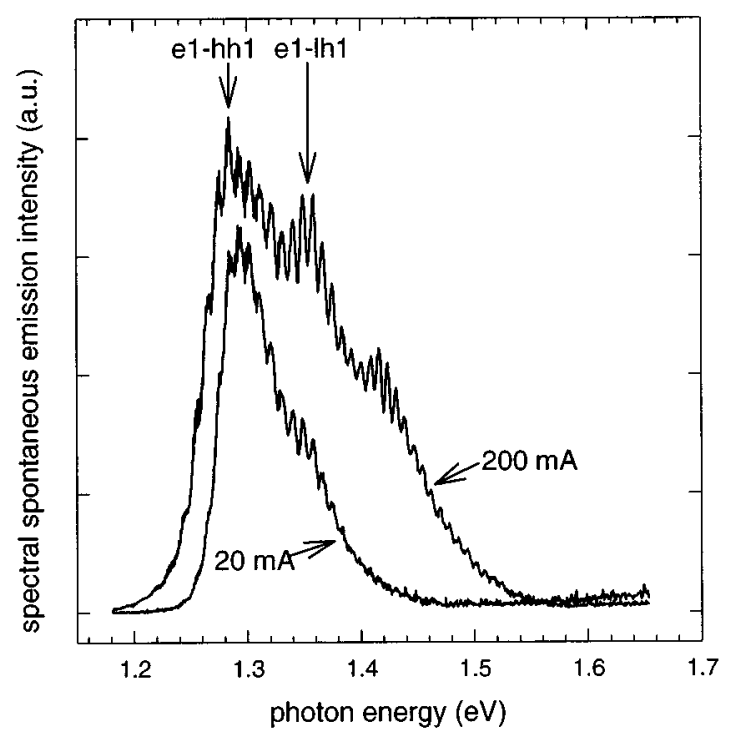

FIG. 1. Normalized spectra at the laser midpoint for currents below and above the $50 \mathrm{~mA}$ threshold.

energy spacing of $0.009 \mathrm{eV}$, suggesting a resonance between the cleaved edge and the ridge. The period of oscillation was invariant under translation of the fiber.

The evolution of spectral shape with increasing current exhibited in Fig. 1 motivated our exploration of the development of theoretical transition peak intensities with increasing carrier density. If a fixed relation was found to exist between the intensity of one of the transition peaks and the carrier density in the active region in the operating range of the laser, then the shape of the carrier density distribution could then be mapped out experimentally. Spontaneous emission spectra were therefore calculated ${ }^{9}$ for the quantum-well system under study, and the evolution with carrier density of the peaks associated with the allowed electron-heavy-hole transitions was followed since these were most easily discerned in the measured spectra. Figure 2 shows the calculated relationship between the transition peak intensities and the carrier density. The intensity of the lowest-energy transition is very nearly linear with carrier density up to $N=3 \times 10^{18}$ $\mathrm{cm}^{-3}$ because the hole and electron densities of states see the approximately linear portion of the Fermi distribution function near the Fermi energy. At low carrier densities, the second electron-heavy-hole transition peak lies in the exponential tail of the Fermi distribution function, giving rise to exponential growth with carrier density at these energy levels. Only when the quasi-Fermi-level separation approaches the energy of this higher transition does an approximately linear relation take over.

Proceeding further with modeling, we compared the temperature sensitivity of the peak-following technique proposed herein with that of the fixed photon energy approach. ${ }^{8}$ We did this by imposing a linear increase in temperature with carrier density up to a typical $60{ }^{\circ} \mathrm{C}$ above the zero-bias temperature at $N=3 \times 10^{18} \mathrm{~cm}^{-3}$, and assuming a rate of band-gap shrinkage of $0.325 \mathrm{meV} /{ }^{\circ} \mathrm{C}$. ${ }^{10}$ The peak-following technique exhibits a slight deviation from linearity due to the

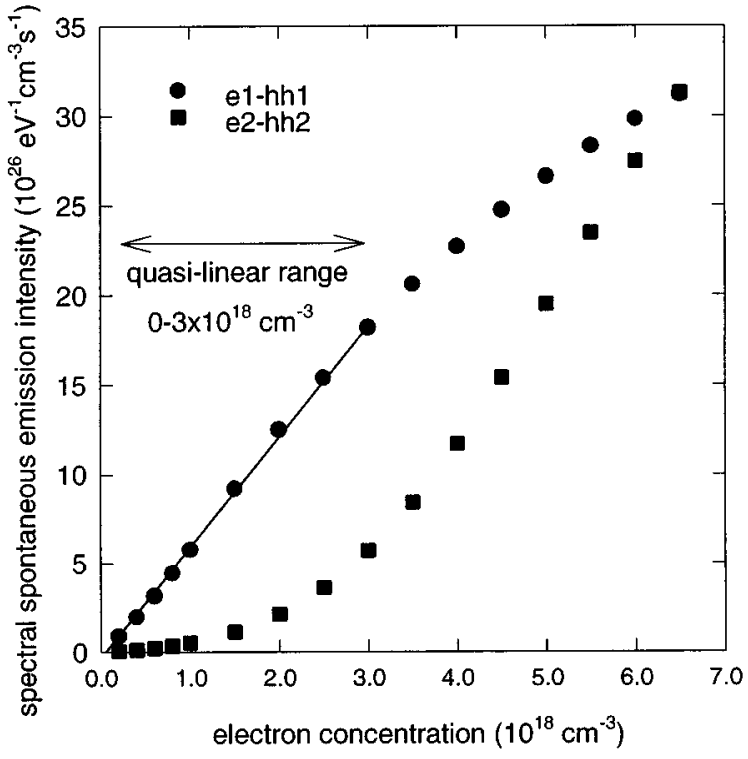

FIG. 2. Theoretical prediction (Ref. 9) of the evolution with carrier density of the amplitude of the first two allowed electron-heavy-hole transition peaks identified in the spontaneous emission spectrum, along with the line of best fit over the linear range.

spreading of the spontaneous emission spectrum as a result of the broadened Fermi function at the higher temperature. In the fixed photon energy approach, because the band gap shifts with temperature, the measured intensity is no longer on the spectral peak, and the peak intensity rolls off more rapidly with increasing carrier density.

It was concluded from our modeling efforts that the carrier density could reasonably be taken to be proportional to the intensity of the first spontaneous emission peak over the regime of operation of the laser, making it possible to study experimentally the development of the longitudinal distribu-

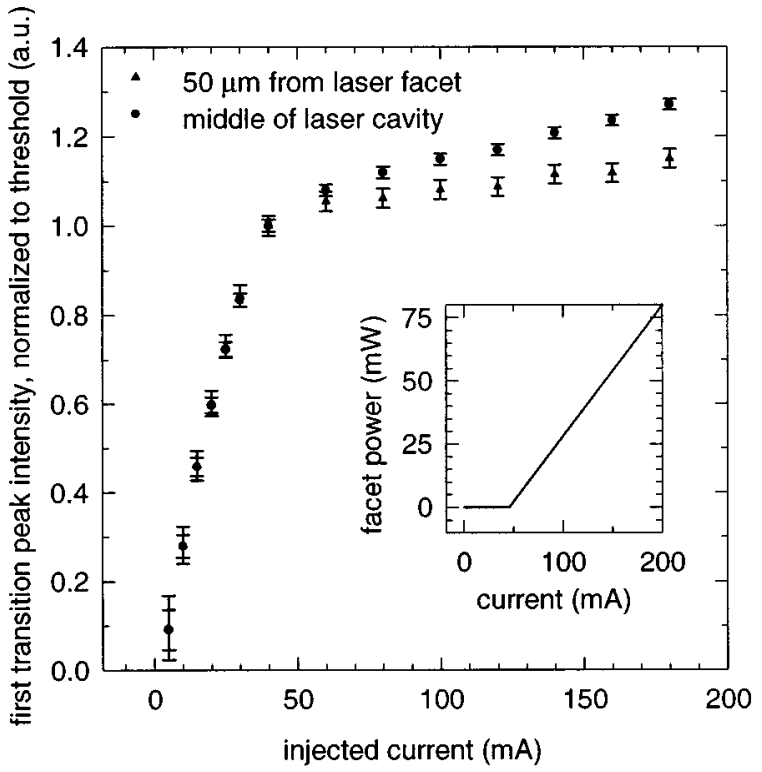

FIG. 3. Experimental peak intensity variation with injected current at midpoint and facet. 


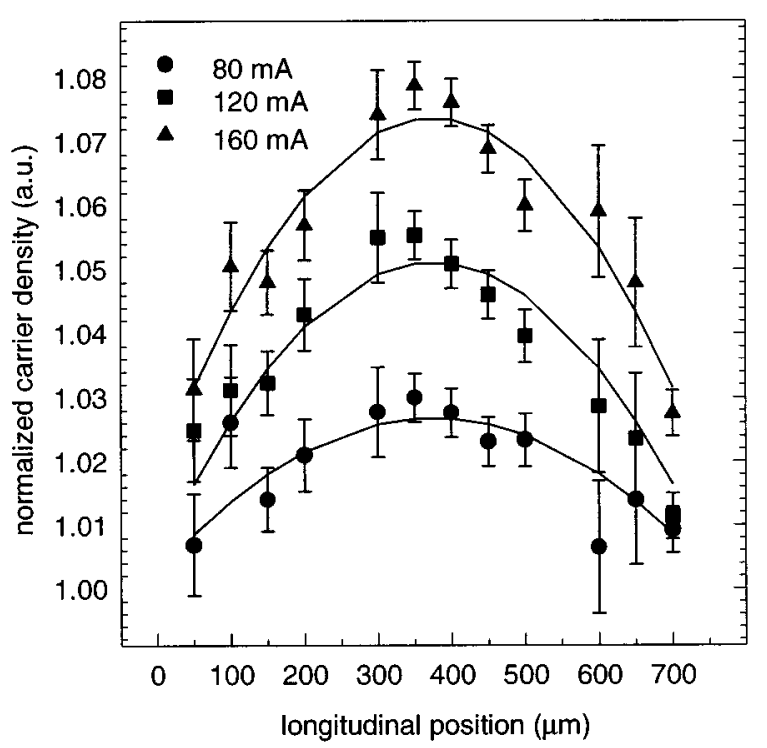

FIG. 4. Profile of peak spontaneous emission intensity normalized to threshold value at each position for a 750- $\mu$ m-long symmetric-cavity laser with $10 \%$ facet reflectivities. Spectra acquired very near the facets (at 0 and 750 $\mu \mathrm{m})$ were dominated by scattered stimulated emission and are not shown. Because of cleave irregularities near the 200 and $550 \mu \mathrm{m}$ positions, a very low signal was obtained at these points, and they are omitted as well.

tion of carriers with current. In Fig. 3 we present a comparison of the rate of growth of the measured spectral spontaneous emission at the peak energy with increased drive current at the midpoint and the facet of a symmetric cavity (10\% reflectivities) laser. Peak intensities at each position are separately normalized to the threshold value at that position. At the facet, the nearly flat relationship between peak intensity and current above threshold indicates carrier pinning. The more prominent growth in peak intensity with current at the laser midpoint reflects a continued increase in carrier density. In Fig. 4 we plot the carrier density profile along with theoretical distributions obtained through self-consistent modeling using a transfer-matrix technique. ${ }^{11}$ The evolution of the shape of the longitudinal carrier density profile with increased current is thus clearly seen. The observed nonclamping is attributable to carrier heating, which causes a decrease in the gain with an increase in the injected current, to the variation in the lateral mode profile which results from carrier-induced changes in refractive index, and to the dependence of gain on the photon density via the nonlinear gain coefficient. $^{5}$

In sum, we have demonstrated an experimental technique for profiling the distribution of carriers in a semiconductor laser. Because of the chosen geometry of our experiment, we are able to recognize and follow specific radiative transition peaks. Our technique is less sensitive than a fixed photon energy approach to band-gap narrowing due to changes in temperature and carrier densities. Our method lends itself to the study of longitudinal distributions of carriers in semiconductor lasers because of the good degree of linearity in the relation between the measured peak intensity and the local carrier density in the typical regime of operation. We are in the process of applying this technique to the study of distributed feedback lasers, in which theoretical predictions of the longitudinal carrier and field intensity profiles are of great interest, but remain to be confirmed experimentally.

${ }^{1}$ B. Hakki and T. Paoli, J. Appl. Phys. 46, 1299 (1975).

${ }^{2}$ C. H. Henry, R. A. Logan, and F. R. Merritt, J. Appl. Phys. 51, 3042 (1980).

${ }^{3}$ K. Tanaka, N. Kotera, and H. Nakamura, Appl. Phys. Lett. 68, 649 (1996).

${ }^{4}$ A. A. Bernussi, H. Temkin, D. L. Coblentz, and R. A Logan, IEEE Photon. Technol. Lett. PTL-7, 348 (1995).

${ }^{5}$ F. Girardin, G.-H. Duan, C. Chabran, P. Gallion, M. Blez, and M. Allovon, IEEE Photon. Technol. Lett. PTL-6, 894 (1994).

${ }^{6}$ P. Blood, E. D. Fletcher, K. Woodbridge, and M. Vening, Appl. Phys. Lett. 57, 1482 (1990).

${ }^{7}$ H. Summers, P. Mogensen, P. Rees, and P. Blood, in Proc. CLEO, CThF4, 1994.

${ }^{8}$ W. W. Fang, C. G. Bethea, Y. K. Chen, and S. L. Chuang, J. Sel. Topics Quantum Electron. 1, 117 (1995).

${ }^{9}$ M. L. Xu, G. L. Tan, J. M. Xu, M. Irikawa, H. Shimizu, T. Fukushima, Y. Hirayama, and R. S. Mand, IEEE Photon. Technol. Lett. PTL-7, 947 (1995).

${ }^{10}$ G. P. Agrawal and N. K. Dutta, Semiconductor Lasers (Van Nostrand, New York, 1993).

${ }^{11}$ H. Anis, T. Makino, and J. M. Xu, IEEE Photon. Technol. Lett. PTL-7, 232 (1995). 